\title{
Influence of Different Levels of Rosemary Leafs Powder Supplementation on Some Productive Traits of Broiler Chicken Ross Strain
}

\author{
Mohammed Jard Kadhim \\ Animal Production Technique Department/ Al musiab Technical College/ AL- Furat AL-Awsat \\ Technical University, Babylon/Iraq \\ mmmedm@yahoo.com
}

Submission date:- 8/3/2018

Acceptance date:- 3/4/2018

Publication date:- 29/8/2018

Keywords: Broiler, Rosemary, Productive traits.

\begin{abstract}
The research was conducted to investigate the effect of different levels of rosemary powder supplementation on some productive traits of broiler chicken ross strain. Three hundred unsexed broiler chicks of one-day old were equally divided into five groups, 2 replicates per group (30 chicks per replicate). Chicks were fed on starter diet from one-day old until end of three week then replaced to finisher diet from the beginning of four week until the end of six week, starter and finisher diet supplemented with four ratio from rosemary powder $0,0.5,1,1.5$ and $2 \%$ which represented experimental groups.

Results showed a significant superiority $(\mathrm{P} \leq 0.05)$ in average live body weight, weight gain, feed conversion ratio and dressing percentage in birds supplemented with rosemary powder as compared with control group at 3 and 6 week of birds age. Moreover supplementation of rosemary had significantly $(\mathrm{P} \leq 0.05)$ decreased mortality ratio in experimental groups as compared with control group.
\end{abstract}

It can be concluded that supplementation of rosemary powder to diet had a profound effect to enhance productive traits of broiler chicken ross strain.

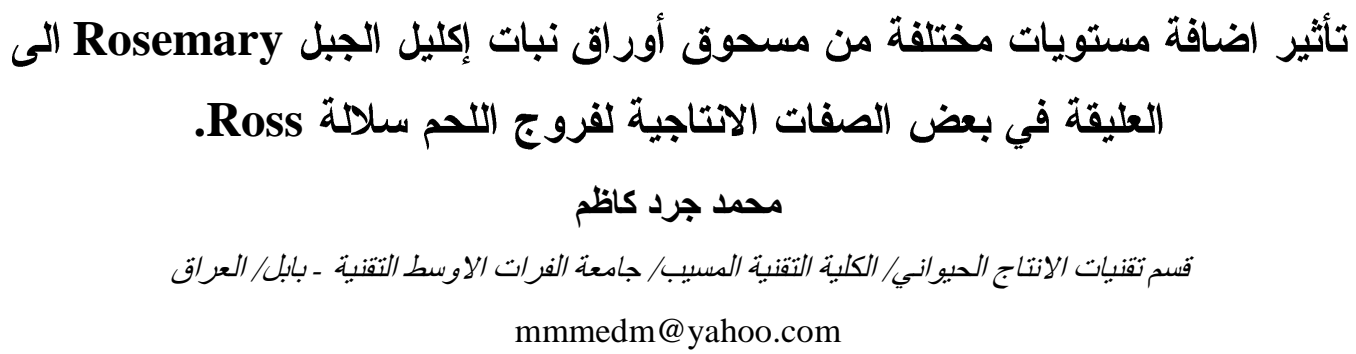

أجري هذا البحث بإضافة مستويات مختلفة من مسحوق أوراق نبات إكليل الجبل الى العليقة بهدف دراسة تأثير ها في بعض الصفات

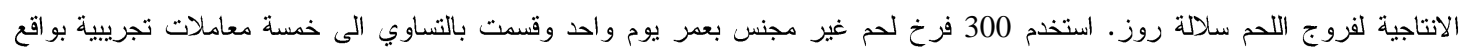
مكررين لكل معاملة (30 فرخ لكل مكرر), غذيت الافراخ على عليقة بادئ من عمر يوم واحد الى نهاية الاسبوع الثالث بعدها استبدلت بعليقة

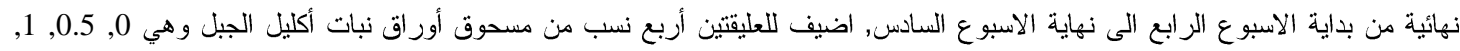

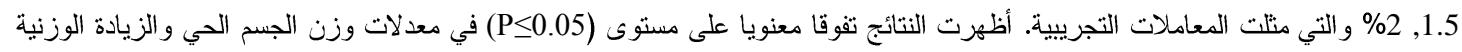

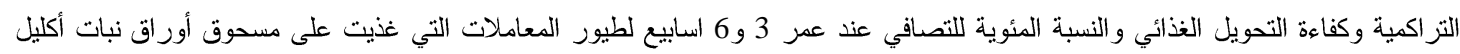

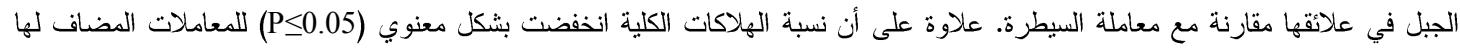
مسحوق أور اق نبات إكليل الجبل مقارنة مع معاملة السيطرة. 
نستتج من هذه الدراسة أن اضافة مسحوق أوراق نبات إكليل الجبل الى العليقة له تأثير ايجابي في تحسين الصفات الانتاجية لفروج

Ross اللحم سلالة.

الكلمات الدالة: فروج اللحم, إكليل الجبل, الصفات الانتاجية.

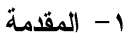

ان الهدف الاساسي لتزبية الطيور الداجنة هو الحصول على منتجات ذات قيمة غذائية عالية متمتلة في البيض واللحم اللذين يعدان من

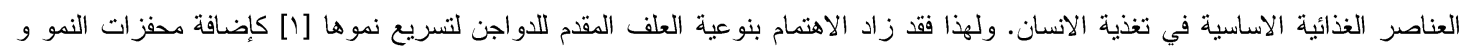
بعض المضادات الحيوية Antibiotics مما انعكس سلبا على صحة الانسان [r] الامر الذي دفع الباحثين لإيجاد مواد طبيعية بديلة لهذه الاضافات

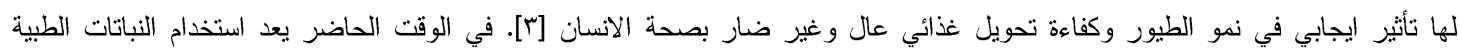

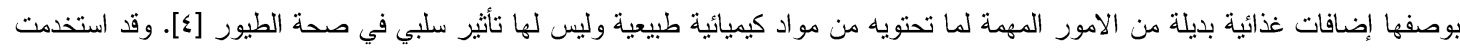
النباتات الطبية أو مستخلصاتها في علائق الدواجن بوصفها مضادات طبيعية للأحياء المجهرية ومحفزات نمو طبيعية تعمل على بلى تحسين الصفات

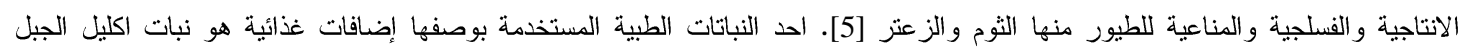
Rosemary

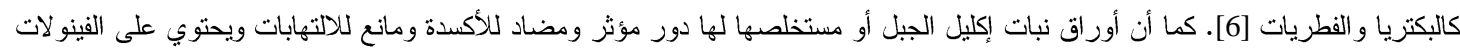

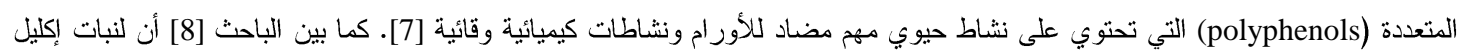

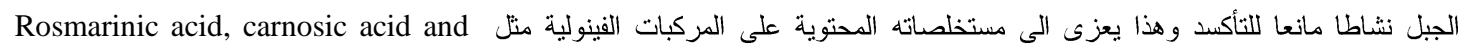
(carnosol) إن الدراسة الحالية تهدف الى معرفة تأثير اضافة مستويات مختلفة من مسحوق أوراق نبات أكليل الجبل Rosemary الى عليقة فروج

اللحم في تحسين بعض الصفات الانتاجية.

r- r r المواد وطر ائق العمل

اجريت هذه التجربة في حقل الطيور الداجنة التابع لقسم تقنيات الانتاج الحيواني/ الكلية التقنية المسيب/ جامعة الفرات الاوسط التقنية خلال

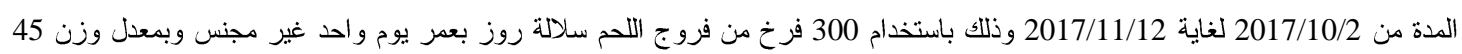

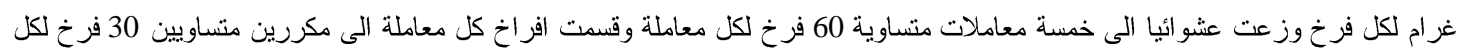
مكرر. احتوت التجربة على المعاملات الأتية:-

المعاملة الاولى (T1) الطيور فيها غذيت على عليقة بدون أي اضافة (معاملة السيطرة). المعاملة الثانية (T2) الطيور فيها غذيت على عليقة السيطرة مع اضافة مسحوق اوراق نبات اكليل الجبل بنسبة 0.5\% من اليوم الاول لغاية عمر ·6 6 6المعاملة الثالثة (T3) الطيور فيها غذيت على عليقة السيطرة مع اضافة مسحوق أوراق نبات اكليل الجبل بنسبة 1\% من اليوم الاول لغاية عمر 6 6

المعاملة الرابعة (T4) الطيور فيها غذيت على عليقة السيطرة مع اضافة مسحوق أوراق نبات اكليل الجبل بنسبة 1.5\% من اليوم الاول لغاية عمر 6 اسابيع. المعاملة الخامسة (T5) الطيور فيها غذيت على عليقة السيطرة مع اضافة مسحوق أوراق نبات اكليل الجبل بنسبة 2\% من اليوم الاول لغاية عمر 6

تم تتظيف وتعقيم القاعة والادوات المستعملة فيها (المناهل والمعالف والحاضنات والاجهزة الاخرى) باستعمال محلول مخفف من الديتول

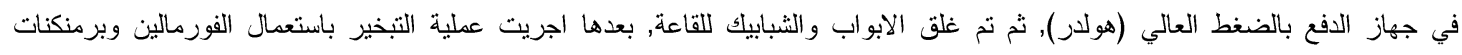

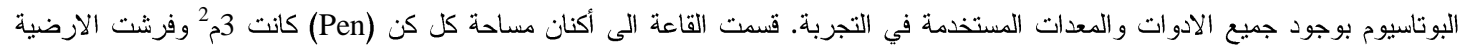
بنشارة الخشب بسمك 3-5 سمع. 
بعد وصول الافر اخ الى القاعة وزعت عشو ائيا على خمسة معاملات بو اقع مكررين لكل معاملة واعطيت ماء مضافا له سكر بمعدل 5 كغم/ 100التز ماء, واستعملت اطباق العلف الدائرية في الاسبوع الاول من التجربة بعدها استبدلت بمعالف معلقة من بداية الاسبوع الثاني. كان

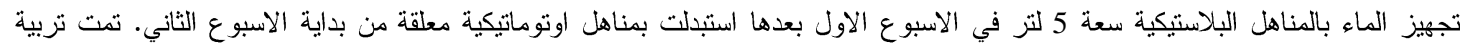
افر اخ فروج اللحم وفق الظروف المثلى الملائمة من حيث برنامج اضاءة مستمر ودرجة حرارة مثالية حيث استخدمت حاضنتين غازيتين لتوفير

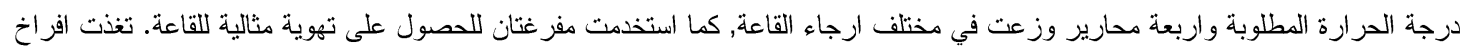

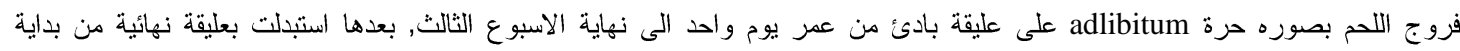
الاسبوع الر ابع اللى نهاية الاسبوع السادس ويوضح الجدول (1) مكونات العليقتين المستخدمنين في التجربة والتحليل الكيمياوي لها.

تم الحصول على أوراق نبات اكليل الجبل الذي استخدم في هذه التجربة من السوق المحلية وتم طحنه بمطحنة كهربائية والنبات المطحون

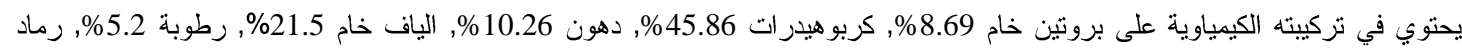

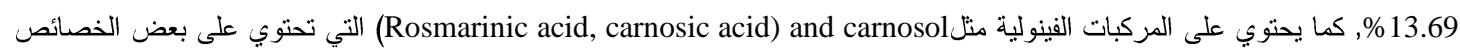

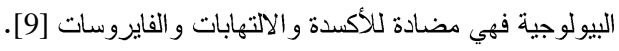
في هذه التجربة تم دراسة بعض الصفات الانتاجية لفروج اللحم حيث تم حساب معدل وزن الجسم الحي ومعدل الزيادة الوزنية واستهلالك العلف ومعامل التحويل الغذائي والنسبة المئوية للتصافي والنسبة المئوية للهلاكات.

تم تحليل بيانات التجربة باستعمال التصميم العشوائي الكامل Completely Randomized Design-CRD لدراسة تأثير المعاملات المختلفة في الصفات المدروسة, وقورنت الفروق المعنوية بين المتوسطات باختبار دنكن متعدد الحدود [10]. واستخدم برنامج [11] في التحليل الاحصائي حسب الانموذج الرياضي الأتي: YIJ=M+TI+EIJ حيث ان : قيمة الصفة المدروسة, M المتوسط العام للصفة المدروسة, ت تأثثر المعاملة حيث تضمن البحث خمسة معاملات, TI EIJ

جدول (1) نسب المواد العلفية الداخلة في التجربة (البادئ و النهائية) و التحليل الكيمياوي لها

\begin{tabular}{|c|c|c|}
\hline 4 عليقة نهائية & 1 يو عليقة بادئ & المادة العلفية \\
\hline 19 & 18 & حنطة \\
\hline 44 & 43 & ذرة صفر اء \\
\hline 22.5 & 26 & كسبة فول الصويا \\
\hline 10 & 10 & *مركز بروتيني لحم \\
\hline 3.5 & 2.0 & زيت نباتي \\
\hline 0.4 & 0.4 & حجر كلس \\
\hline 0.4 & 0.4 & ملح طعام \\
\hline 0.1 & 0.1 & 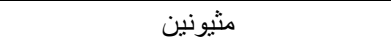 \\
\hline 0.1 & 0.1 & لايسين \\
\hline \multirow[t]{2}{*}{100} & 100 & المجموع \\
\hline & & **التحليل الكيمياوي المحسوب \\
\hline 20.69 & 22.18 & البروتين الخام \% \\
\hline 3100.4 & 2968.01 & الطاقة الممثلة (كيلو سعرة / كيلو غر ام) \\
\hline 149.85 & 133.81 & نسبة الطاقة الممثلة / البروتين الخام \% \\
\hline
\end{tabular}


Journal of University of Babylon for Pure and Applied Sciences,Vol.(26), No.(8): 2018

\begin{tabular}{|c|c|c|}
\hline 1.16 & 1.27 & اللايسين \% \% \\
\hline 0.82 & 0.86 & المثيونين + السستين \% \\
\hline 1.03 & 1.02 & كالسيوم \% ك \\
\hline 0.47 & 0.45 & فسفور \% \\
\hline 5.01 & 6.02 & الياف خام \% \\
\hline 3.27 & 3.23 & 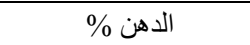 \\
\hline
\end{tabular}

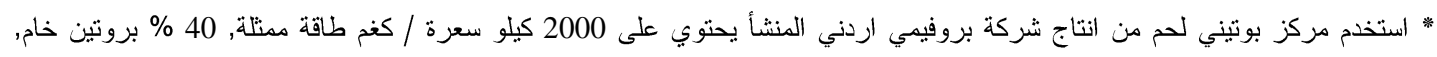

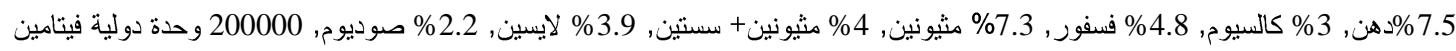

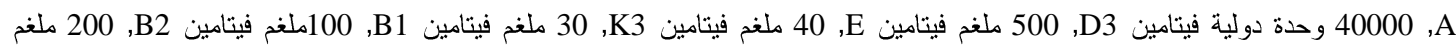

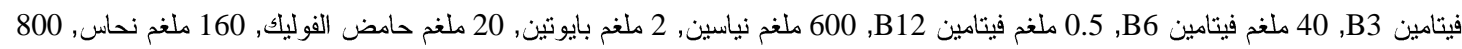
ملغم حديد, 1200 ملغم منغنيز , 1000 ملغم زنكائ, 8 ملغم يود. "* حسبت كل من البروتين والطاقة الممثلة و اللايسين و المثيونين+ الستثين و الكالسيوم والفسفور والالياف والدهن لكل مادة علفية حسب [12].

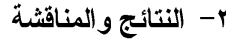

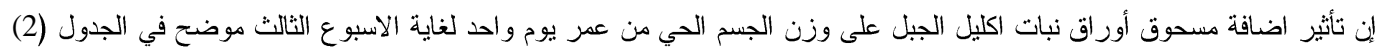

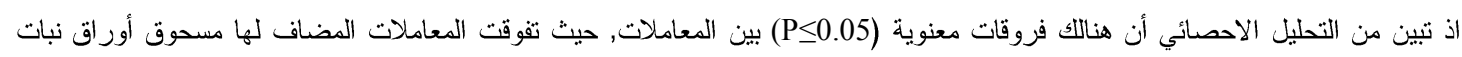

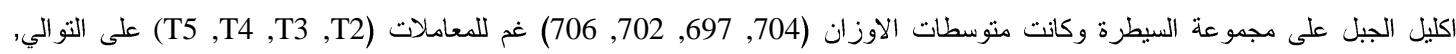

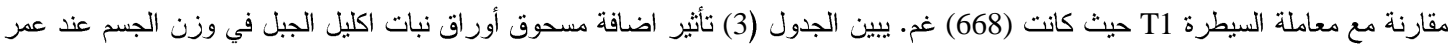

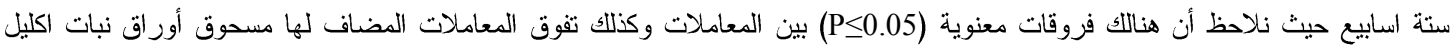

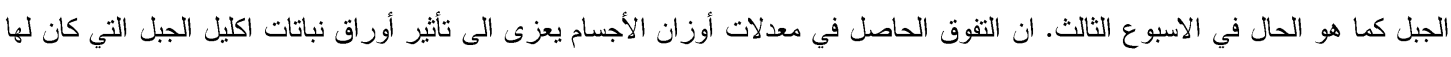

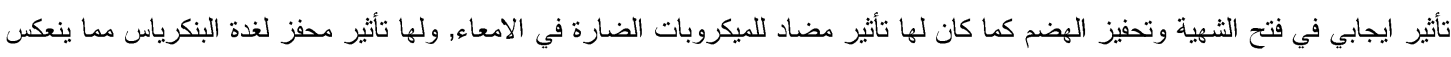

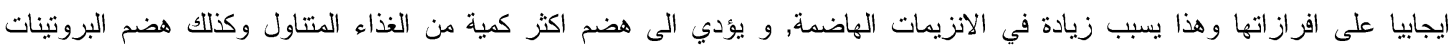

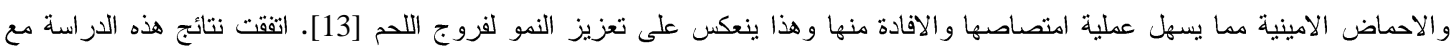

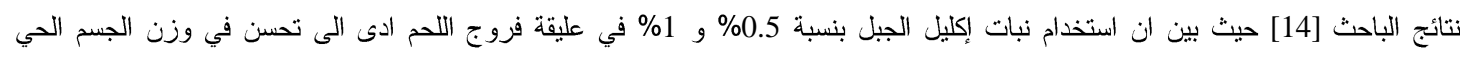
اللطيور عند كلى النسبتين وبمستوى معنوية (PS0.05) مقارنة مع مجموعة السيطرة.

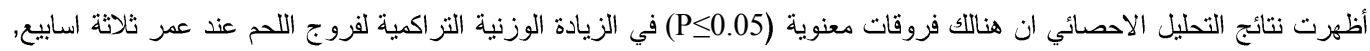

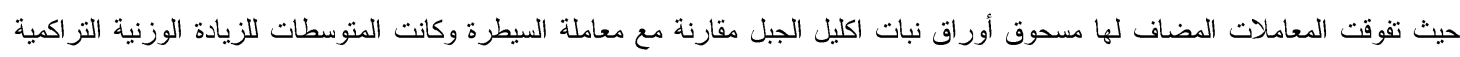

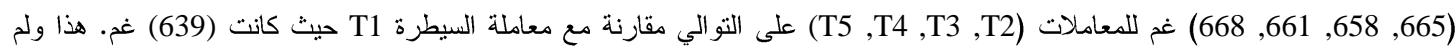

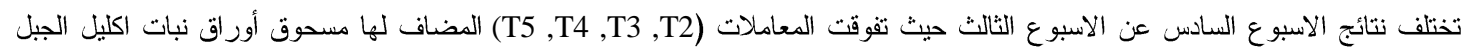

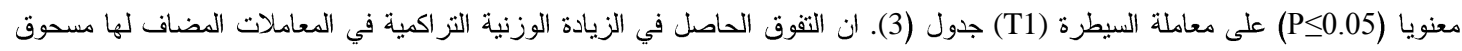

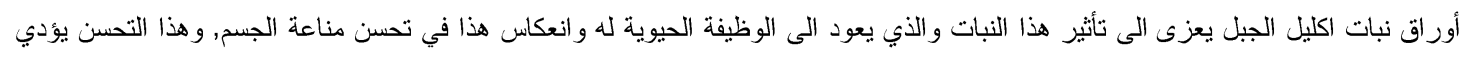

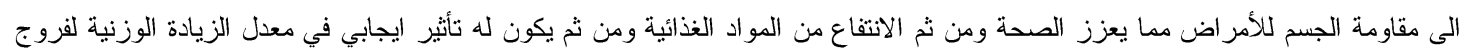

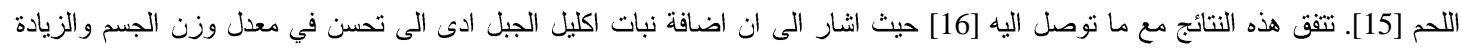
الوزنية لفروج اللحم.

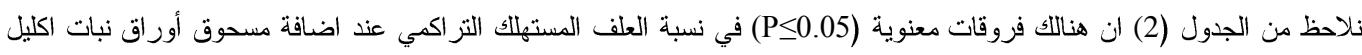

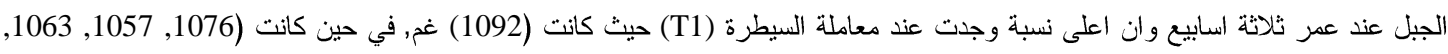

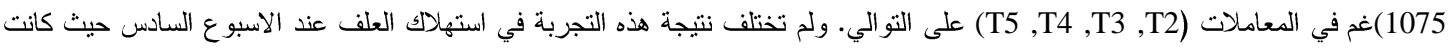

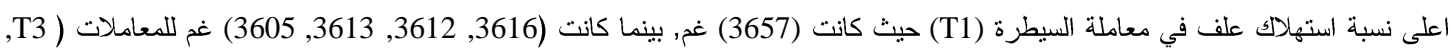

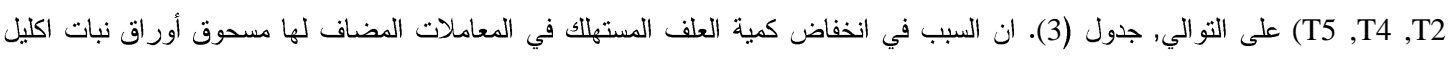
الجبل يعود الى تحسن قابلية الهضم لاى الطيور لما تحتويه هذه النباتات من احماض فينولية تمنلك خصائص مضادة للأحياء المجهرية الضارة 
وهي تحسن الحالة الصحية للطيور ومن ثم الانتفاع التام من المواد العلفية [17]. إن نتائج هذه الدراسة مماتلة لما توصل اليه الباحثان [18] اللذان

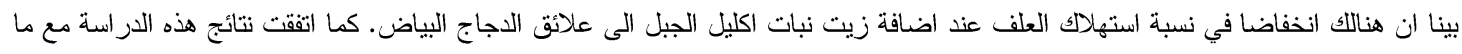

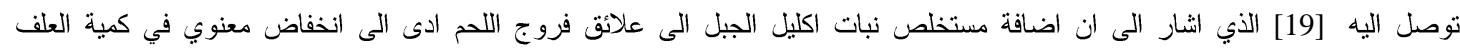
المستهرلك.

جدول (2) تأثير اضافة مسحوق أوراق نبات اكليل الجبل الى العلف في وزن الجسم الحي والزيادة الوزنية التزراكية واستهلاك العلف التزراكمي ومعامل التحويل الغذائي لفروج اللحم عند عمر ثلاثة اسابيع.

\begin{tabular}{|c|c|c|c|c|}
\hline \multicolumn{4}{|c|}{ المتوسط د الخطأ القياسي } & \multirow[b]{2}{*}{ المعاملات } \\
\hline / معامل التحويل الغذائي (غم علف وزنية) & نسبة العلف المستهلك التر اكمي & 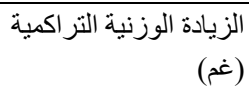 & (غم) معدل وزن الجسم & \\
\hline $1.70 \pm 0.026$ & $1092 \pm 11.22$ & $639 \pm 4.201$ & $668 \pm 4.237$ & $\mathrm{~T} 1$ \\
\hline $\mathrm{a}$ & $\mathrm{a}$ & $\mathrm{b}$ & $\mathrm{b}$ & \\
\hline $1.61 \pm 0.019$ & $1076 \pm 10.76$ & $665 \pm 4.113$ & $704 \pm 4.532$ & $\mathrm{~T} 2$ \\
\hline $\mathrm{b}$ & $\mathrm{b}$ & $\mathrm{a}$ & $\mathrm{a}$ & \\
\hline $1.60 \pm 0.016$ & $1057 \pm 12.28$ & $658 \pm 3.927$ & $697 \pm 3.867$ & $\mathrm{~T} 3$ \\
\hline $\mathrm{b}$ & $b$ & $\mathrm{a}$ & $\mathrm{a}$ & \\
\hline $1.60 \pm 0.012$ & $1063 \pm 10.88$ & $661 \pm 4.746$ & $702 \pm 4.361$ & $\mathrm{~T} 4$ \\
\hline $\mathrm{b}$ & $\mathrm{b}$ & a & $\mathrm{a}$ & \\
\hline $1.60 \pm 0.014$ & $1075 \pm 11.54$ & $668 \pm 3.657$ & $706 \pm 4.795$ & T5 \\
\hline $\mathrm{b}$ & $\mathrm{b}$ & $\mathrm{a}$ & $\mathrm{a}$ & \\
\hline$*$ & $*$ & $*$ & $*$ & مستوى المعنوية \\
\hline
\end{tabular}

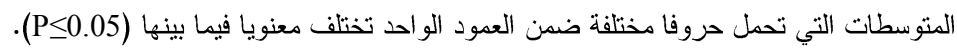

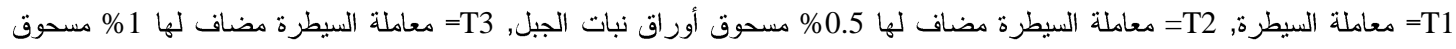

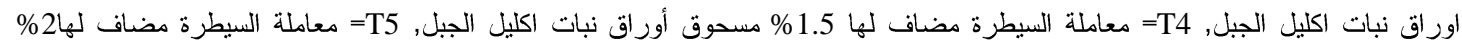
مسحوق أوراق نبات اكليل الجبل.

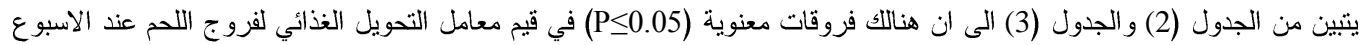

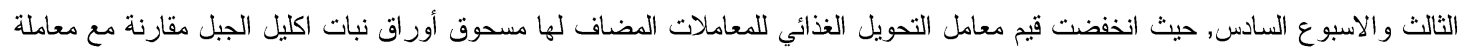
السيطرة, وان انخفاض هذه القيم يدل على وجود تحسن في تحويل العلف المتتاول الى وزن جسم حي. يعود هذا التحسن في معامل التحويل

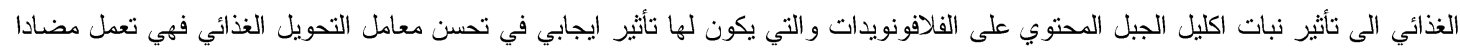

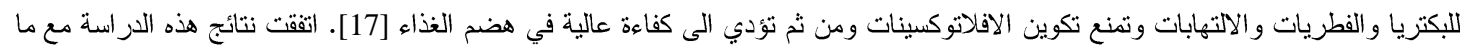

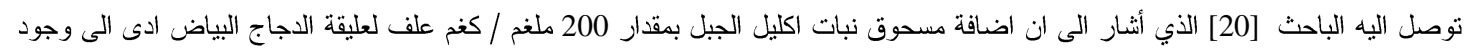
تحسن في قيمة معامل التحويل الغذائي مقارنة بمجمو عة السيطرة. جدول (3) تأثير اضافة مسحوق اوراق نبات اكليل الجبل الى العلف في وزن الجسم الحي و الزيادة الوزنية التزاكمية واستهلاك العلف التزاكمي ومعامل التحويل الغذائي لفروج اللحم عند عمر ستة اسابيع. 
Journal of University of Babylon for Pure and Applied Sciences,Vol.(26), No.(8): 2018

\begin{tabular}{|c|c|c|c|c|}
\hline \multicolumn{4}{|c|}{ المتوسط 土 الخطأ القياسي } & \multirow[b]{2}{*}{ المعاملات } \\
\hline مع زيادل التحويل الغذائي (غم علف / & نسبة العلف المستهلك التر اكمي & 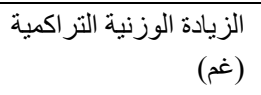 & معدل وزن الجسم (غم) & \\
\hline $\begin{array}{c}1.84 \pm 0.012 \\
\mathrm{a}\end{array}$ & $\begin{array}{c}3657 \pm 5.22 \\
\text { a }\end{array}$ & $\begin{array}{c}1984 \pm 4.20 \\
\mathrm{c}\end{array}$ & $\begin{array}{c}1920 \pm 3.601 \\
\mathrm{c}\end{array}$ & $\mathrm{T} 1$ \\
\hline $\begin{array}{l}1.74 \pm 0.09 \\
b\end{array}$ & $\begin{array}{l}3616 \pm 6.11 \\
b\end{array}$ & $\begin{array}{c}2076 \pm 5.13 \\
\text { b }\end{array}$ & $\begin{array}{c}2122 \pm 2.537 \\
b\end{array}$ & $\mathrm{~T} 2$ \\
\hline $\begin{array}{l}1.73 \pm 0.06 \\
b\end{array}$ & $\begin{array}{l}3612 \pm 5.02 \\
\quad b\end{array}$ & $\begin{array}{c}2078 \pm 4.72 \\
b\end{array}$ & $\begin{array}{c}2214 \pm 3.267 \\
\mathrm{a}\end{array}$ & $\mathrm{T} 3$ \\
\hline $\begin{array}{l}1.74 \pm 0.011 \\
b\end{array}$ & $\begin{array}{l}3613 \pm 4.88 \\
\mathrm{~b}\end{array}$ & $\begin{array}{c}2072 \pm 4.76 \\
\text { b }\end{array}$ & $\begin{array}{c}2118 \pm 4.161 \\
b\end{array}$ & $\mathrm{~T} 4$ \\
\hline $\begin{array}{l}1.71 \pm 0.04 \\
b\end{array}$ & $\begin{array}{c}3605 \pm 6.15 \\
c\end{array}$ & $\begin{array}{c}2099 \pm 3.57 \\
\text { a }\end{array}$ & $\begin{array}{c}2219 \pm 4.795 \\
a\end{array}$ & T5 \\
\hline$*$ & $*$ & * & $*$ & مستوى المعنوية \\
\hline
\end{tabular}

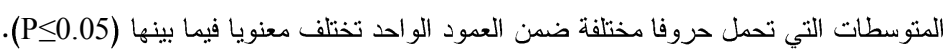

مع=T2 معاملة السيطرة, =T2 معاملة السيطرة مضاف لها 0.5\%مسحوق أوراق نبات الجيل, T3= معاملة السيطرة مضاف لها1 \% مسحوق

أوراق نبات اكليل الجبل, T4= معاملة السيطرة مضاف لها 1.5\% مسحوق أوراق نبات اكليل الجبل, T5= معاملة السيطرة مضاف لهاف

2\%مسحوق أوراق نبات اكليل الجبل.

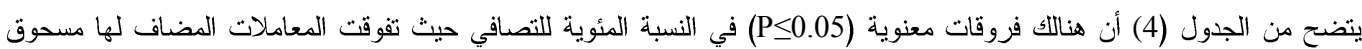

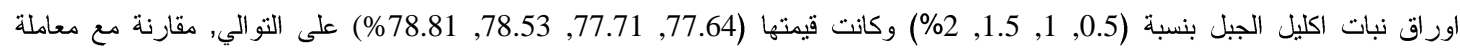
السيطرة حيث كانت (74.87). ويعزى التفوق الحاصل في نسبة التصافي المعاملات المضاف لها مسحوق أوراق نبات اكليل الجبل الى التأثير

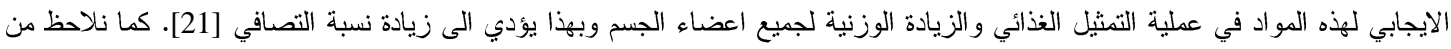

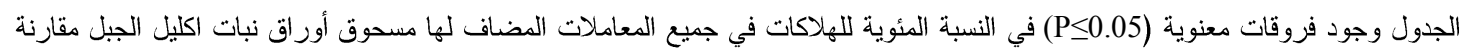

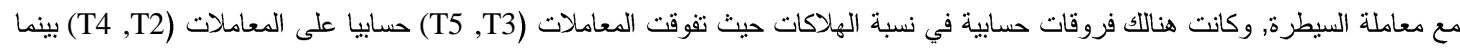

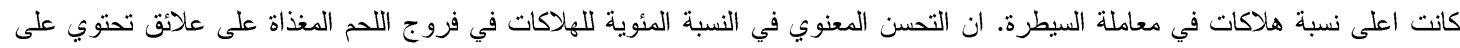

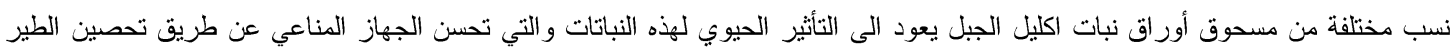

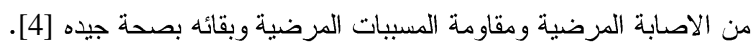
جدول (4) تأثير اضافة مسحوق اوراق نبات اكليل الجبل الى العلف في النسبة المئوية للتصافي والنسبة المئوية للهلاكات لفروج اللحم عند عمر ستة اسابيع.

\begin{tabular}{|c|c|c|}
\hline \multicolumn{2}{|c|}{ المتوسط د الخطأ القياسي } & \multirow[b]{2}{*}{ المعاملات } \\
\hline النسبة المئوية للهلاكات & النسبة المئوية للتصافي & \\
\hline $\begin{array}{c}6.00 \pm 4.00 \\
b\end{array}$ & $\begin{array}{c}74.87 \pm 0.60 \\
b\end{array}$ & $\mathrm{~T} 1$ \\
\hline $\begin{array}{c}2.00 \pm 5.00 \\
\mathrm{a}\end{array}$ & $\begin{array}{c}77.64 \pm 0.57 \\
\mathrm{a}\end{array}$ & $\mathrm{T} 2$ \\
\hline $\begin{array}{c}0.00 \pm 0.00 \\
\mathrm{a}\end{array}$ & $\begin{array}{c}77.71 \pm 0.67 \\
\mathrm{a}\end{array}$ & T3 \\
\hline $\begin{array}{c}2.00 \pm 4.00 \\
\mathrm{a}\end{array}$ & $\begin{array}{c}78.53 \pm 0.61 \\
\mathrm{a} \\
\end{array}$ & $\mathrm{T} 4$ \\
\hline $\begin{array}{c}0.00 \pm 3.57 \\
\mathrm{a}\end{array}$ & $\begin{array}{c}78.81 \pm 0.54 \\
\mathrm{a}\end{array}$ & T5 \\
\hline$*$ & $*$ & مستوى المعنوية \\
\hline
\end{tabular}




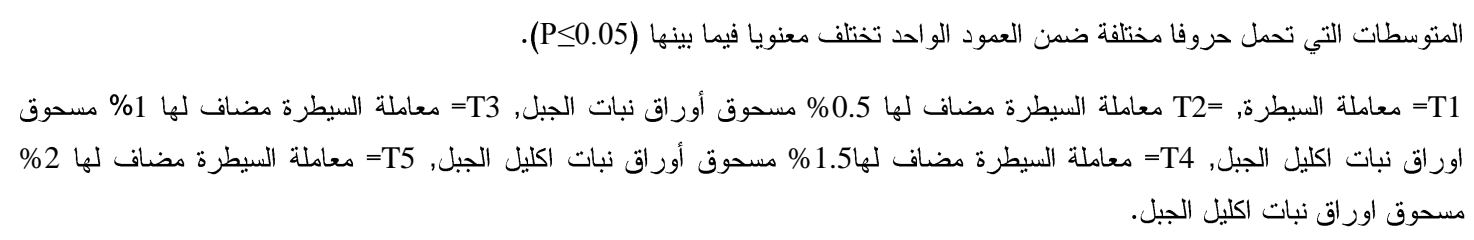

CONFLICT OF INTERESTS

There are no conflicts of interest.

\section{4- References}

[1] USDA, "Additives in Meat and Poultry Products ", Apr. 10, 2008. [Online]. Available: http://www.fsis.usda.gov/factsheets/Additives_in_Meat_\&_Poultry_Products/index.asp [Accessed: Apr. 10, 2008].

[2] B. Revington, "Feeding poultry in the post antibiotic era“, Multi- State Poult. Meeting May 14-16. pp. 1-14, 2002.

[3] L. Peric, D. Zikic, M. Lukic, "Aplication of alternative growth promoters in broiler production ", Biotechnology in Animal Husbandry, 25, 387- 397, 2009.

[4] M. Osman, H. M. Yakout, H. F. Mot Awe, and W. F. Ezz ElAlarb, "Productive, physiological, immunological and economical effect of supplementing natural feed additives to broiler ", Egypt. Poult. Sci. 30 (1), 25-53, 2010.

[5] A. Amouzmehr, D. Behrouz, G. N. Jalil, Il. S. Kyung, L. Jayant, and F. Fereidoun, "Effect of garlic and thyme extracts on growth performance and carcass characteristics of broiler chicks" , J. Sci. and Technology, 54 (3), 185190, 2012.

[6] D. Bown, "New encyclopedia of herbs and their uses" , The royal horticultural society. London, New york, Melbourne, Delhi, 2002.

[7] S. Cheung, and J. Tai, "Anti-proliferative and antioxidant properties of rosemary (Rosmarinus officnalis) ", Oncology Report. 17, 1525-1531, 2007.

[8] N. Troncoso, H. Sierra, L. Carvajal, P. Depliano, and G. Gunther, "Fast high performance ultraviolet visible quantification principle phenolic antioxidants in liquid chromatography and fresh rosemary ", J. chromat. 1100, 20-50, 2005.

[9] S. J. Posadas, V. Caz, C. Largo, "Protective effect of supercritical fluid rosemary extract, Rosmarinus officnalis, on antioxidants of major organs of age rats ", Exp Gerontol, 44, 383-389, 2009.

[10] D. B. Duncan, "Multiple range and multiple F-tests. Biometrics", 11, 1-42, 1955.

[11] SAS, "SAS User's Guide“, Statistics Version. 7.0, SAS Institute, Inc. Cary, NC. USA, 2004.

[12] NRC. National Research Council,"Nutrient requirements of poultry National academy of science “, Washington, DC, 1994.

[13] F. Hernandez, J. Madrid, V. Garcia, J. Orengo, and M. D. Meglas, "Influence of two plants extracts on broiler performance, digestibility and digestive organs size “, Poult. Sci. 83,169-174, 2004.

[14] G. A. M. Al-Kassie, "The effect of anise and rosemary on broiler performance" , Int. J. Sci. 7(3), 243-245, 2008.

[15] K. Trianaphyhou, G. Blekas, and D. Boskou, "Anti-oxidative properties of water extracts obtained from herbs of the spices lamiaceae “, International Journal of Food Sciences and Nutrition. 52 (4), 313-317, 2001.

[16] R. J. Abbas, "Effect of using fenugreek, parsley and sweet basil seeds as feed additives on the performance of broiler chickens”, Int. J. Poult. Sci. 9 (3), 278-282, 2010.

[17] C. J. Lopez -Bote, J. I. Gray, E. A. Gomaa, and C. J. Flegal, 'Effect of dietary administration of oil extracts from rosemary and sage on lipid oxidation in broiler meat “, Br. Poult. Sci. 39, 235-240, 1998.

[18] P. Williams, and R. Losa, "The use of essential oils and their compounds in poultry nutrition ", World poult. 17 (4), 14-15, 2001.

[19] S. K. Ashan, "Influence of two herbs extract o performance, carcass quality and blood parameters in broiler chicken", Annals of biological research, 2 (5), 584-588, 2011.

[20] S. B. Canan, E. M. Kuddusi, and O. Kaynar, "The effect on feeding thyme, sage and rosemary oil on laying hen performance, cholesterol and some proteins ratio of egg yolk and Escherichia coli count in feces", Aech. Geflugelk. 72 (5), 231-237, 2008.

[21] S. Mellor, "Nutraceuticals- alternatives to antibiotics“, World Poult. 16, 30-33, 2000. 